\title{
Plasmid Segregation- Maintenance of Bacterial Replicons
}

\author{
Nida Tabassum Khan *
}

Department of Biotechnology, Faculty of Life Sciences and Informatics, Balochistan University of Information Technology Engineering and Management Sciences, (BUITEMS), Quetta, Pakistan

*Corresponding author: Nida Tabassum Khan, Department of Biotechnology, Faculty of Life Sciences and Informatics, Balochistan University of Information Technology Engineering and Management Sciences, (BUITEMS), Quetta, Pakistan, Tel: +03368164903; E-mail: nidatabassumkhan@yahoo.com

Received: August 02, 2017; Accepted: August 25, 2017; Published: September 05, 2017

Citation: Khan NT (2017) Plasmid Segregation- Maintenance of Bacterial Replicons. Ann Clin Lab Res Vol.5:No.3:187.

\section{Abstract}

Plasmid segregation is a means of bacterial plasmid replication through which identical copies of plasmids are produced and is equally distributed among the newly produced daughter cells. Segregation mechanisms such as site-specific, active partition, toxin/anti-toxin system ensures that plasmid copy number is under control and is stably inherited.

Keywords: Site- specific recombination; Active partition system; Toxin/anti-toxin system

\section{Introduction}

Segregation generates exact copies of the extra circular plasmid and ensures their stable distribution to the newly produces daughter cells during bacterial binary fission [1]. In case when plasmid stable-state copy number is increased then the passive diffusion ensures stable transfer of a single copy of the plasmid to each of the daughter cells produced during fission in order to keep plasmid copy number count under the normal levels. However it is still suggested that random diffusion is adequate for the stable transmission of plasmids keeping their copy number count from moderate to high. But such plasmids were not freely distributed rather sorted into subcellular regions for equal distribution directed by an unfamiliar mechanism [2,3].

\section{Literature Review}

\section{Plasmid segregation is by means of the following three ways}

- Site-specific recombination

- Active partition system

- Toxin and anti-toxin system

Site specific recombination: Site-specific segregation occurs at a precise position in the nucleotide sequence of the DNA. This recombination mechanism was first reported in $E$. coli in which DNA integration was observed between $E$. coli and $\lambda$ [4]. DNAs of both organisms possessed a specific attachment site of specific nucleotide sequences (5'-TTTATAC-3') which permits both the DNAs to base pair together. Then after attachment integrase catalyzes the two single strand disruptions. After a short branch migration, the integrase exerts a second strand cuts on two other strands [5].

Active partition system: Active partitioning segregation mechanism localized the plasmids in the bacterial cell in such a way that after cell division both of the daughter cells gains a copy of the plasmid [6].

Example- P1 plasmid active partition mechanism in E. coli. The partition system is structured in a cassette that comprises of an auto-regulated operon containing genes parA and parB and parS cis-acting sequence placed downstream [7]. Both the genes encode proteins which interacts with the host proteins and integration factor to produce a nucleoprotein aggregate at parS which interacts with an unfamiliar partitioning complex of the host. This complex directs the bound copies of P1 plasmid to the one-quarter and three-quarter cell-length positions following replication at the mid-cell. As the bacterial cell lengthens, the plasmids remain at these positions. As the cell divides at its midpoint the plasmids occupy the same positions of the new cells and replication and partition cycles are repeated. This mechanism of segregation is quite common in low-copy-number bacterial plasmids, toxin and anti- toxin system $[8,9]$.

Toxin and anti- toxin system: Toxin-antitoxin (TA) systems are pairs of genes that encode a stable and unstable toxic peptide and an antitoxin (either protein or an antisense RNA) respectively. The prime purpose of plasmid toxin-antitoxin system is to steady the plasmid by eradicating daughter cells that are plasmid deficient by a mechanism known as post segregation killing. These systems are widespread in numerous bacteria but have not reported in pathogen Campylobacter jejuni that causes human gastroenteritis [10].

Examples: 1) In $\mathrm{F}$ plasmid encoded $\mathrm{CcdAB}$ toxin-antitoxin system, DNA gyrase poison a $\mathrm{CcdB}$ toxin traps a cleavage complex between gyrase and DNA and allies with DNA gyrase to form an aggregate that is compromised in supercoiling activity. The consequences of such disruptions are fatal for $E$. 
coli [11]. 2) pVir plasmid encoded toxin-antitoxin system of Campylobacter jejuni.

The antitoxin is a small 101 nucleotide non-translated antisense RNA called Campylobacter jejuni RNA antitoxin (cjrA). This non-translated antisense RNA is complementary to the $3^{\prime}$ region of the mRNA that encodes Campylobacter jejuni. Peptide toxin (CjpT), a toxic peptide. Cloning and expression of cjpT gene alone in Escherichia coli inhibited bacterial growth. Inactivation of CjpT in C. jejuni resulted in rapid loss of pVir plasmid during in vitro passage. The presence of the cjrA small RNA in $C$. jejuni was demonstrated by Northern blotting. Cloning of an extra copy of cjrA into the wild-type $C$. jejuni in-trans caused depletion of pVir, further confirming its antitoxic function. qRT-PCR demonstrated that the expression level of the cjpT gene was influenced by RNase III (rnc) as the transcript levels of CjpT was consistent among different growth phases in wild type strain, but had dynamic changes in there mutant background. Together, these results establish that cjpT and cjrA encode a functional toxin-antitoxin system that is required for the stability of the pVir plasmid in $C$. jejuni [12].

A number of diverse toxin-antitoxin systems are widely distributed on bacterial plasmids but the intracellular targets for the toxin components of these systems probably varies. Toxin-antitoxin homologs have also been reported on bacterial DNA responsible for initiating programmed cell death in bacteria during unfavorable environmental conditions. High and low-copy-number plasmids often make use of active partition and toxin-antitoxin systems to promote stable plasmid segregation $[13,14]$.

\section{Plasmid distribution among bacterial populations}

Few bacterial strains possess natural competency for transformation by absorbing the extra circular fragment of DNA or by means of bacteriophage mediated DNA transduction [15]. However, the most common mechanism of DNA transfer reported in majority of bacterial population is through conjugation between a donor and recipient cells. Plasmids involved in conjugation DNA transfer confers antibiotic resistance, bacteriocin production and have been well recognized in Archaea and Eubacteria [16]. Moreover, such plasmid could be transferred among distantly related divergent Gram-negative and Gram-positive Eubacteria, and even from Eubacteria to yeast and bacteria to plant hosts [17].

\section{Plasmid evolution}

Plasmids are among the key modules in the evolutionary diversification of prokaryotes and their adaptation to the inconsistent environmental conditions [18]. They constitute with the dispensable genome of the prokaryotes i.e., nonessential regions of the chromosomes and contributes in various auxiliary activities. Stereotypically, a plasmid consists of one or more essential genes encoding proteins involved in recombination functions along with genes directing a diverse array of accessory components that functions in different metabolic mechanisms. Such DNA sequences are not encoded by the organism nuclear genome [19]. Essentially, plasmid genes are truly under the influence of variance selection recurrently losing and gaining genes with the passage of time revealing the flexible structural construct of the plasmid. This flexibility is chiefly due to the lavishness of mobile genetic transposable elements they have which enables generation of homologous regions by specific intra- and intermolecular recombination or by means of horizontal and vertical gene transfer. These events increase the prospect that similar plasmids can exists in different hosts' genome enhancing their genes organization and reshuffling of their functions [20].

\section{Discussion}

Regardless of their contributions in prokaryotic evolution, still little is revealed due to lack of extensive correspondences between them with the exception for DNA sequences involved in duplication and recombination which obscure phylogenetic analyses based on gene descent and synteny [21,22]. In order to solve this problem to some extend Blast2 network, a bioinformatics approach is used to provide an immediate conception of the similarities, existing among DNA and protein sequences based on similarity networks rebuilding and phylogenetic profiling. This will in turn, opens the possibility to trace the evolutionary aspects and origin of the entire plasmids and not only of single genes and/or operons harbored by them [23].

\section{Conclusion}

Thus, the above-mentioned segregation mechanisms of plasmid ensure maintenance of plasmid copy number to normal levels and their inheritance to the bacterial progeny.

\section{References}

1. Gordon GS, Sitnikov D, Webb CD, Teleman A, Straight A, et al. (1997) Chromosome and low copy plasmid segregation in E. coli: visual evidence for distinct mechanisms. Cell 90: 1113-1121.

2. Ebersbach G, Gerdes K (2005) Plasmid segregation mechanisms. Annu Rev Genet 39: 453-479.

3. Gordon S, Rech J, Lane D, Wright A (2004) Kinetics of plasmid segregation in Escherichia coli. Molecular Microbiol 51: 461-469.

4. Fondi M, Bacci G, Brilli M, Papaleo MC, Mengoni A, et al. (2010). Exploring the evolutionary dynamics of plasmids: The Acinetobacter pan-plasmidome. BMC Evol Biol 10: 59.

5. Zhang L, Ou X, Zhao G, Ding X (2008) Highly efficient in vitro sitespecific recombination system based on Streptomyces phage фBT1 integrase. J bacteriol 190: 6392-6397.

6. Jensen MJ, Jensen RB, Löwe J, Gerdes K (2002) Prokaryotic DNA segregation by an actin like filament. The EMBO Journal 21: 3119-3127.

7. Salje J, Gayathri P, Löwe J (2010) The ParMRC system: Molecular mechanisms of plasmid segregation by actin-like filaments. Nature Reviews Microbiol 8: 683.

8. Williams DR, Thomas CM (1992) Active partitioning of bacterial plasmids. Microbiol 138: 1-16. 
9. Funnell BE (2005) Partition-mediated plasmid pairing. Plasmid 53: 119-125.

10. Yamaguchi Y, Park JH, Inouye M (2011) Toxin-antitoxin systems in bacteria and archaea. Annu Revi Genet 45: 61-79.

11. Rawlings DE (1999) Proteic toxin-antitoxin, bacterial plasmid addiction systems and their evolution with special reference to the pas system of pTF-FC2. FEMS Microbiol Lett 176: 269-277.

12. Mayer MP, Bueno LC, Hansen EJ, Di Rienzo JM (1999) Identification of a cytolethal distending toxin gene locus and features of a virulence-associated region in Actino bacillus actinomycetemcomitans. Infection and immunity 67: 1227-1237.

13. Magnuson RD (2007) Hypothetical functions of toxin-antitoxin systems. J Bacteriol 189: 6089-6092.

14. Hayes F (2003) Toxins-antitoxins: Plasmid maintenance, programmed cell death, and cell cycle arrest. Science 301: 1496-1499.

15. Thomas CM, Nielsen KM (2005) Mechanisms of and barriers to, horizontal gene transfer between bacteria. Nature reviews. Microbiol 3: 711.
16. Davison J (1999) Genetic exchange between bacteria in the environment. Plasmid 42: 73-91.

17. Sørensen SJ, Bailey M, Hansen LH, Kroer N, Wuertz S (2005) Studying plasmid horizontal transfer in situ: A critical review. Nature Reviews Microbiol 3: 700.

18. Cohen SN (1976) Transposable genetic elements and plasmid evolution. Nature 263: 731-738.

19. Bouma JE, Lenski RE (1988) Evolution of a bacteria/plasmid association. Nature 335: 351-352.

20. Davison J (1999) Genetic exchange between bacteria in the environment. Plasmid 42: 73-91.

21. Modi RI, Adams J (1991) Coevolution in bacterial plasmid populations. Evolution 45: 656-667.

22. Eberhard WG (1990) Evolution in bacterial plasmids and levels of selection. Quart Rev Biol 65: 3-22.

23. Brilli $M$, Mengoni A, Fondi $M$, Bazzicalupo $M$, Liò $P$, et al. (2008) Analysis of plasmid genes by phylogenetic profiling and visualization of homology relationships using Blast2 Network. BMC Bioinformatics 9: 551. 